\title{
Glueball and hybrid mass and decay with string tension below Casimir scaling
}

\author{
Elsa Abreu ${ }^{1}$ and Pedro Bicudo ${ }^{2}$ \\ ${ }^{1}$ Dep. Física, Instituto Superior Técnico, Av. Rovisco Pais, 1049-001 Lisboa, Portugal \\ 2 Dep. Física and CFTP, Instituto Superior Técnico, Av. Rovisco Pais, 1049-001 Lisboa, Portugal \\ E-mail: elsabreu@netcabo.pt and bicudo@ist.utl.pt
}

Received 30 October 2006

Published 4 December 2006

Online at stacks.iop.org/JPhysG/34/195

\begin{abstract}
Lattice computations with excited $S U(3)$ representations suggest that the confining gluon-gluon interaction complies with the Casimir scaling. The constituent gluon models have also assumed the Casimir scaling. Nevertheless, inspired by type-II superconductors, we explore a new scenario for the gluongluon interaction where the adjoint string is replaced by a pair of fundamental strings, resulting in a factor of 2, smaller than the Casimir scaling factor of 9/4. To test our proposal we construct a simple constituent gluon model, extrapolated from the funnel potential for quarkonium, and apply it to compute the wavefunction of glueballs and of hybrid gluelumps. From the decay widths of quarkonium, we also extrapolate the decay widths of the glueballs. Our predictions apply to charmonia, lightonia, glueballs and hybrid gluelumps with large angular momentum $J$.
\end{abstract}

Communicated by Professor S Kabana

\section{Introduction}

Glueballs are expected from QCD, and indeed they are observed in pure gauge lattice QCD simulations. The first experimental evidence for glueballs is an indirect one, the pomeron Regge trajectory, which explains the high energy scattering of hadrons [1-4]. Systematic comparisons of the glueball masses computed in lattice QCD with the pomeron trajectory, initiated by Llanes-Estrada et al, confirm that the lattice QCD glueballs comply with the pomeron [4-7]. Direct experimental evidence for glueballs is still controversial [8], nevertheless in a few years two major collaborations will update the experimental search for glueballs, PANDA at GSI and GLUEX at JLAB. Different theoretical approaches to glueballs are trying to match the experimental effort. 
(a)

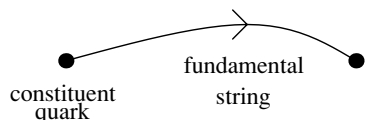

quark

string

(b)

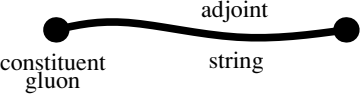

(c)

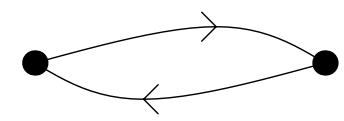

(d)

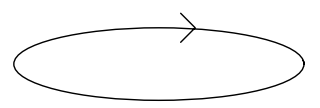

Figure 1. Basic models (b), (c) and (d) for glueballs, extended from the string model (a) for quark-antiquark confinement in a meson.

Table 1. Experimental (review of particle physics) available masses. $M$ and widths $\Gamma$, for charmonia, lightonia, $\mathrm{K}^{*}$, glueballs and gluelumps, with maximal $s$ and $j=l+s$.

\begin{tabular}{|c|c|c|c|c|c|c|}
\hline Family $\backslash J^{\mathrm{PC}}$ & $0^{++}$ & $1^{--}$ & $2^{++}$ & $3^{--}$ & $4^{++}$ & $5^{--}$ \\
\hline Charmonium, $M$ & & 3096 & 3556 & - & - & - \\
\hline Charmonium, $\Gamma$ & & 0.1 & 2.1 & - & - & - \\
\hline Lightonium $I=0, M$ & & 783 & 1275 & 1667 & 2034 & - \\
\hline Lightonium $I=0, \Gamma$ & & 8.5 & 185 & 168 & 222 & - \\
\hline Lightonium $I=1, M$ & & 775 & 1318 & 1689 & 2010 & - \\
\hline Lightonium $I=1, \Gamma$ & & 150 & 107 & 161 & 353 & - \\
\hline Lightonium $\mathrm{K}^{*}, M$ & & 892 & 1430 & 1776 & 2045 & - \\
\hline Lightonium $\mathrm{K}^{*}, \Gamma$ & & 51 & 105 & 159 & 198 & - \\
\hline Glueball, $M$ & & & - & & - & \\
\hline Glueball, $\Gamma$ & & & - & & - & \\
\hline Gluelump, $M$ & & & - & - & - & - \\
\hline Gluelump, $\Gamma$ & & & - & - & - & - \\
\hline
\end{tabular}

Here we study a new simple and plausible model for glueballs, and apply it to hadrons with maximal angular momentum $J$. These large $J$ states are particularly interesting because they determine the lower Regge trajectories in Chew-Frautschi plots. They also have simpler wavefunctions, with no radial excitations and with a clearly dominant angular and spin wavefunction, and therefore they may be easier to identify experimentally. The maximal $J$ states are also appealing theoretically because the string length is large and the details of the confining string become relevant. The experimentally confirmed resonances only go up to $J=4$ for light mesons and $J=2$ for charmonium (see table 1), therefore most of our results are predictions. Hopefully some of these large $J$ hadrons will be observed in the future experiments.

To extend to glueballs the confining string mechanism, we first discuss quark mass generation and quark-antiquark confinement. The quark model describes most of the hadronic phenomenology, simply assuming constituent quarks, a short range coulomb interaction and a long range confining string interaction. This model is depicted in figure 1(a). Nambu and Jona-Lasinio [9] showed that the massless fermions can have a mass generated by the 
spontaneous breaking of chiral symmetry, with the Schwinger-Dyson technique, equivalent to a second-quantized canonical transformation [10-12]. Indeed this has been successfully applied to quark models [10-12], suggesting that quark models may comply both with the PCAC theorems and with the success of quark model spectroscopy [13-16]. Moreover, the quark-antiquark confining string, consisting of a colour-electric flux tube, was suggested by Nielsen and Olesen [17]. Nielsen and Olesen conjectured that the magnetic flux tube vortex of type-II superconductors could be extended to colour-electric flux tube strings in QCD.

Inspired in the constituent string-confined quark model (a), three models for glueballs are plausible. They are respectively depicted in figure 1. In model (b), a constituent gluon pair is confined with an adjoint string, in model (c), a constituent gluon pair is confined with a pair of fundamental strings and in model (d), a closed fundamental string loop is rotating, without any constituent gluon at all. Models (b) [3, 4, 18-20], with a pair of constituent gluons confined by an open adjoint string, and (d) [21,22], with a closed fundamental string and no constituent gluon, and also hybrid models $[23,24]$, have been studied in the literature. The model (c) is explored in this paper.

In this paper we specialize in the proposed model (c), extending the basic constituent quark model, from the quark-antiquark meson, to the gluon-gluon glueball and to the quark-antiquark-gluon hybrid. Because we also study light mesons, our kinetic energy is relativistic. Our specialization in large $J$ states also allows us to avoid the details of spintensor potentials, and we will not compute the spin-tensor potentials for quarks and gluons here.

In section 2 we motivate and discuss the parameters of our model. The scaling of 2, the experimental meson masses, the glueball masses and parameters obtained in lattice QCD and in the Schwinger-Dyson equations, together with the pomeron intercept determine all the parameters of the model. In section 3 we compute the masses (up to $5 \mathrm{GeV}$, close to the energy limit of the future experiments) and the mean radius of charmonia, lightonia, glueballs and hybrids. We also estimate decay constants in section 4 , from the breaking of the fundamental. In section 5 we conclude.

\section{The parameters of this constituent quark and gluon model}

\subsection{Motivating the double fundamental string}

An important assumption of models (b) and (c) is the massive constituent gluon. There is evidence for a massive-like dispersion relation for the gluon, with a mass ranging from $700 \mathrm{MeV}$ to $1000 \mathrm{MeV}$, both from lattice QCD [25-28] and from Schwinger-Dyson equations [29]. This suggests that the Anderson-Higgs mechanism is providing a consistent framework for the mass generation of gauge bosons. For instance in the Meissner effect of superconductors, the photon has a mass. It is plausible that simple non-relativistic constituent gluon models may indeed describe glueballs.

The next important ingredient is the gluon-gluon interaction. Like in the quark model, the two contributions for the gluon-gluon interaction are the shorter range one-gluon exchange interaction, proportional to a QCD Casimir invariant $\lambda^{a} \lambda^{a}$, and the longer range confining string interaction, proportional to the colour-electromagnetic linear energy density of the string.

Model (b) considers the adjoint string interaction, also known as the Casimir scaling interaction. The adjoint string is a natural extension of the fundamental string assumed in models (a), (c) and (d). It consists of an excited string with a colour-electric flux larger than that of the fundamental string. In the fundamental representation of $S U(3)$, acting in the quark 
colour triplet, the Lie algebra is represented by the $3 \times 3$ Gell-Mann fundamental matrices $\lambda^{a}$. The product of the fundamental $\lambda^{a}$ is

$$
\lambda^{a} \lambda^{b}=\frac{2}{3} \delta^{a b} I+d^{a b c} \lambda^{c}+\mathrm{i} f^{a b c} \lambda^{c},
$$

where the antisymmetric structure constants $f^{a b c}$ are antisymmetric and independent of the representation. The constant tensors $\frac{2}{3} \delta^{a b}$ and $d^{a b c}$ are symmetric and depend on the representation. Another relevant representation is the adjoint representation. In the adjoint representation, acting in the gluon colour octet, the Lie algebra matrices are represented by the $8 \times 8$ adjoint matrices,

$$
\left[\lambda_{\text {adjoint }}^{a}\right]_{b c}=2 \mathrm{i} f^{b a c} \text {. }
$$

With equation (1), and using the properties of the antisymmetric $f^{a b c}$ and of the symmetric $d^{a b c}$ we find that the Casimir invariant in the fundamental and adjoint representation are respectively,

$$
\lambda^{a} \lambda^{a}=\frac{16}{3} I, \quad \lambda_{\text {adjoint }}^{a} \lambda_{\text {adjoint }}^{a}=12 I,
$$

and they differ by a factor of $9 / 4$, the Casimir scaling. In lattice QCD, Bali showed that the energy of excited colour-electric flux tubes is proportional to the $\lambda^{a} \lambda^{a}$. In pure-gauge lattice QCD, Debbio et al and Bali [30, 31], extended the lattice gauge field links from the fundamental representation to several representations of $S U(3)$, and found that the colourcolour interactions comply with the Casimir scaling. They are all proportional to $\lambda \cdot \lambda$, except for smaller than 5\% numerical errors. This Casimir scaling is the simplest possible result, and Semay [32] showed that it occurs if the colour-electric flux-tube thickness is essentially the same for all possible quantized fluxes.

Nevertheless two fundamental strings cost less energy than one adjoint string. Therefore we propose here a new model (c) with the same pair of constituent gluons of (b), but with a gluon-gluon potential lower [33] than the Casimir scaling of model (c). The scaling of the present model is $2<9 / 4$.

Like the seminal paper of Nielsen and Olesen [17], model (c) is inspired by type-II superconductors $[34,35]$. In type-II superconductors the magnetic flux is allowed to penetrate, and it is localized in vortices, with flux

$$
\phi_{n}=n \frac{h c}{2 e}
$$

where the number $n$ quantifies the magnetic flux. Rather than having a single vortex with a high flux, it is energetically favourable to have several elementary vortices, say a square lattice of vortices, each with the minimal flux $\phi_{1}$. This occurs because the energy of each vortex is proportional to the square of the magnetic flux. For instance, a vortex with $n=2$ does cost the double of the energy of two elementary vortices with $n=1$, while both scenarios have the same flux $\phi_{2}=2 \phi_{1}$.

Notice also that in type-II superconductors the vortices suffer a repulsive interaction, which prevents them from summing to excited vertices. In type-I superconductors, the opposite scenario occurs. The first excited vortex has an energy smaller than the energy of a pair of elementary vortices. And in type-I superconductors the elementary vortices suffer an attractive interaction, which pulls them to form excited vortices.

In what concerns QCD, it is not clear yet if QCD is like a type-I superconductor, in which case model (b) would be the correct one or if it is like a type-II superconductor, in which case model (c) is the correct one. Importantly, model (c) is not excluded by the lattice QCD computations published so far. The Casimir scaling for the octet-octet interaction 
Table 2. Parameters of the constituent model for glueballs, charmonium and gluelumps.

\begin{tabular}{llllll}
\hline Family & $m_{1}(\mathrm{GeV})$ & $m_{2}(\mathrm{GeV})$ & $\alpha$ & $\sigma\left(\mathrm{GeV}^{2}\right)$ & $\mathcal{C}(\mathrm{GeV})$ \\
\hline Charmonium & 1.64 & 1.64 & 0.27 & 0.25 & -0.69 \\
Lightonium & 0.44 & 0.44 & 0.35 & 0.25 & -0.80 \\
Glueball & 0.80 & 0.80 & $2 \times 0.27$ & $2 \times 0.25$ & -0.52 \\
Gluelump & 0.80 & 3.28 & $2 \times 0.27$ & $2 \times 0.25$ & -1.59 \\
\hline
\end{tabular}

was observed in lattice discretizations of QCD where the fundamental links are replaced by adjoint links, $8 \times 8$ matrices of the adjoint representation of $S U(3)$. Therefore these lattice computations are not adequate to simulate our model (c) which assumes fundamental strings only. Baker et al [36, 37], who developed dual QCD, found that dual QCD is close to the frontier between type-I and type-II superconductivity.

Although we do not claim that model (b) is wrong, we submit that model (c) is plausible because it is energetically favourable to have two fundamental strings, with scaling factor of 2 , than one adjoint (excited) string, with a scaling factor of $9 / 4=2.25$. In the remaining of this paper we explore model (c), where the constituent gluons are confined by a pair of fundamental strings.

\subsection{Mesons}

Our starting point to parametrize the potentials and masses is the funnel potential for charmonium [38]. We upgrade the model with relativistic kinetic energies to apply it to large angular excitations. The parameters of this model include the charm $m_{1}$ and anti-charm $m_{2}$ identical masses, and a Coulomb potential, a linear potential and a constant potential,

$$
V(r)=-\frac{\alpha}{r}+\sigma r+\mathcal{C}
$$

The Coulomb potential $-\frac{\alpha}{r}$ can be extracted from the perturbative QCD one-gluon exchange potential and from the non-perturbative Luscher fluctuations of the string [39]. All Coulomb contributions are summed in the parameter $\alpha$. The linear potential is parametrized by the string tension $\sigma$. Both can be extracted from lattice QCD. Moreover the linear potential needs some Coulomb interaction to linearize the Regge trajectory in the Chew-Frautschi plot. A constant $\mathcal{C}$ term in the potential is also needed to fit the spectrum. Here the $\mathcal{C}$ sums the effect of spin-tensor potentials and of vacuum effects. Moreover, in lattice QCD the constant term in the potential is not determined, and in a sense this constitutes a free parameter of the funnel potential. Here we use the parameters $m_{1}=m_{2}, \alpha, \sigma$ and obtained by Lucha and Schöberl [38] to fit the charmonium spectrum. Because our kinetic energy is relativistic, we refit the constant $\mathcal{C}$. Our charmonium parameters are shown in table 2 .

Our next class of hadrons is the lightonia, mesons composed of light quarks. In this case the masses are expected to be slightly larger than the third of the lighter baryon masses (see, for instance, the modelling of baryons by Isgur and Karl [40]). In what concerns the Coulomb and string tension, they may be slightly different from those of charmonium. The parameters that we used to fit the experimental spectrum are again shown in table 2 . While the same charmonium string tension can be used, a larger Coulomb potential is needed to linearize the light meson Regge trajectory, and the constant potential is also slightly refitted. This is consistent with the Fermi-Breit short range Coulomb potential, which increases for light masses. 


\subsection{Glueballs and hybrids}

Our next class of hadrons is that of glueballs. The mass of the constituent gluon is determined from the Schwinger-Dyson equation for the gluon propagator, [29] equivalent to the Bogoliubov-Valation mass gap equation [41] for the gluon propagator, and with lattice QCD computations of the gluon propagator [27, 28]. Although the gluon propagator is not gauge invariant, and it is not clear whether gauge symmetry is broken or not, there is evidence for a mass ranging from $700 \mathrm{MeV}$ to $1 \mathrm{GeV}$ in the dispersion relation of the gluon. This scale is also present in the lattice QCD determinations of the first excitation of the quark-antiquark or three-quark potentials $[42,43]$. Here we use a mass of $800 \mathrm{MeV}$, lighter than the mass of a light quark-antiquark pair. In this case the constituent gluon might be relatively stable against the decay to a quark-antiquark pair. Note that, although in non-dynamical models the mass of light constituent quarks is of the order of $300 \mathrm{MeV}$, in dynamical models, where an attractive funnel potential is used, the constituent quark mass is larger. Here we use a light constituent quark mass of $440 \mathrm{MeV}$ [38], fitting correctly the meson spectrum. Such a large constituent quark mass may also be compatible with the magnetic moment of the ground state baryons, because the quarks have an anomalous magnetic moment driven by spontaneous breaking of chiral symmetry [44].

We already discussed in detail the present model (c) of the double fundamental string, and therefore for the string tension of the glueballs we double the string tension $\sigma$ already used for the quarks.

For the parameter $\alpha$ of the glueball Coulomb potential, a similar factor of 2 can also be used. This can be indirectly verified from the masses of glueballs in lattice QCD. The lattice QCD result for the hyperfine splitting between the masses of the $2^{++}$and $0^{++}$is of the order of $0.7 \mathrm{GeV}$. This splitting can be compared to the hyperfine splitting between the $1^{--}$and $0^{-+}$ mesons, which is of the order of $0.4 \mathrm{GeV}$ for light mesons (once chiral effects are subtracted) and is of the order of $0.1 \mathrm{GeV}$ for charmonium. Using the one-gluon exchange hyperfine splitting potential proportional to $\frac{\alpha \vec{\lambda}_{1} \cdot \lambda_{2} \delta(r)}{m^{2}} \vec{S}_{1} \cdot \vec{S}_{2}$, similar to that derived for mesons [45], Cornwall and Soni [46] and Kaidalov and Simonov [3] found a hyperfine splitting, between the $2^{++}$and $0^{++}$glueballs, of the order of $1.0 \mathrm{Gev}$. Assuming that the hyperfine splitting potential is essentially due to the perturbative one-gluon exchange potential, this suggests that the Coulomb potential should be suppressed by $30 \%$. However, with the scaling factor of 2 , rather than the Casimir scaling of 2.25 , we already decrease the hyperfine potential by a factor of more than $10 \%$. Moreover these authors used gluon masses of the order of 0.5 to $0.6 \mathrm{GeV}$. Our gluon masses are larger (because they are estimated in lattice QCD and in SchwingerDyson truncated QCD equations) and this further decreases the hyperfine potential. Thus we expect that a glueball Coulomb potential with twice the same $\alpha$ already used in charmonium is consistent with a correct glueball hyperfine splitting.

To determine the constant potential $\mathcal{C}$, we fit it with the intercept of the glueball trajectory, which is expected to reproduce the pomeron. The equation for the pomeron trajectory in the $J, t=M^{2}$ space is [47, 48],

$$
\begin{aligned}
& J=\alpha_{p}(t), \\
& \alpha_{p}(t)=1.08+0.25 t .
\end{aligned}
$$

The intercept $\alpha_{p}(t)$ is of the order of 1 , and this explains the high energy hadronic cross sections. In particular, we may ignore the small decimal digits .08 because they may be due to double pomeron exchange. The pomeron is also expected to correspond to a series of glueball masses. Therefore our final parameter $\mathcal{C}$ is fitted to the intercept $\alpha_{p}(0)=1$. Again our glueball parameters are shown in table 2 . 
In what concerns the hybrids, it is possible that we have a gluelump. The charm and anticharm pair may form a colour octet with a mass of $3.3 \mathrm{GeV}$, quite larger than the constituent gluon mass. Then the charm-anticharm octet is essentially stopped at the centre of the mass of the hybrid system, with the glueball orbiting it, attached by a double fundamental string. Such a system of a gluon attached to a heavy colour octet is called a gluelump [49]. Theoretically, this is a very interesting object because it is even simpler than a glueball. All the parameters of the gluelump potential are the same of the glueball potential, except for the constant potential $\mathcal{C}$ which may be refitted.

The constant potential $\mathcal{C}$ is fitted to produce a splitting of $1 \mathrm{GeV}$ between the first gluelump state and the first $S=1$ charmonium state. This splitting has been observed in lattice QCD for quenched quark potentials [42]. Since in the gluelump we ignore the motion of the charm and the anticharm, the constant $\mathcal{C}$ not only accounts for the spin-tensor interaction, and for vacuum effects, it also accounts for the kinetic and potential energy internal to the $c \bar{c}$ pair. Again, our gluelump parameters are shown in table 2.

\section{Masses and radii of glueballs, hybrids and quarkonia with large $J$}

We solve the radial Schrödinger equation, with relativistic kinetic energies (9), with a finite difference method, which transforms the differential equation in a simple matrix eigenvalue method. The parameters of our constituent model for glueballs, charmonium and gluelumps are shown in table 2 .

The charmonium spectrum and wavefunctions are usually determined solving the nonrelativistic radial Schrödinger equation for two-body systems,

$$
\begin{aligned}
& {\left[M_{t} c^{2}-\frac{\hbar^{2}}{2 \mu} \frac{\mathrm{d}^{2}}{\mathrm{~d} r^{2}}+\frac{\hbar^{2}}{2 \mu} \frac{l(l+1)}{r^{2}}+V(r)\right] v_{n l}(r)=E_{n l} v_{n l}(r),} \\
& v_{n l}(r)=r R_{n l}(r), \quad M_{t}=m 1+m 2, \quad \mu=\frac{m_{1} m_{2}}{m_{1}+m_{2}} .
\end{aligned}
$$

However, we are also interested in high angular excitations, and in lighter quark and gluon constituents, and therefore we upgrade the kinetic energy to the relativistic one. In the centre-of-mass frame this amounts to replacing in equation (8),

$$
M_{t} c^{2}+\frac{\mathbf{p}^{2}}{2 \mu} \rightarrow \sqrt{m_{1}^{2} c^{4}+\mathbf{p}^{2} c^{2}}+\sqrt{m_{2}^{2} c^{4}+\mathbf{p}^{2} c^{2}}
$$

Again we can perform the standard angular separation of the Schrödinger equation, where the squared momentum is separated in a simple second derivative and in a centrifugal barrier,

$$
\mathbf{p}^{2} \frac{1}{r} v_{n l}(r) Y_{l}^{m}(\hat{r})=\frac{\hbar^{2}}{r}\left[-\frac{\mathrm{d}^{2}}{\mathrm{~d} r^{2}}+\frac{l(l+1)}{r^{2}}\right] v_{n l}(r) Y_{l}^{m}(\hat{r}),
$$

thus the equation remains a radial equation, the spherical harmonics can be factorized. The arguments of the square roots in equation (9) are positive definite and therefore there is no technical difficulty in computing the relativistic kinetic energy (9). A possible method of finding a function $f(M)$ of a matrix $M$ uses the eigenvalues $\lambda_{i}$ and eigenvectors,

$$
f(M)=\sum_{i}|i\rangle f\left(\lambda_{i}\right)\langle i|
$$

Thus, once the squared momentum in equation (10) is diagonalized, either with spherical Bessel functions or with finite differences, the relativistic kinetic energy operator of equation (9), a function of the squared momentum, can be computed with the method of equation (11). Then we finally solve the Schrödinger equation, diagonalizing the Hamiltonian 


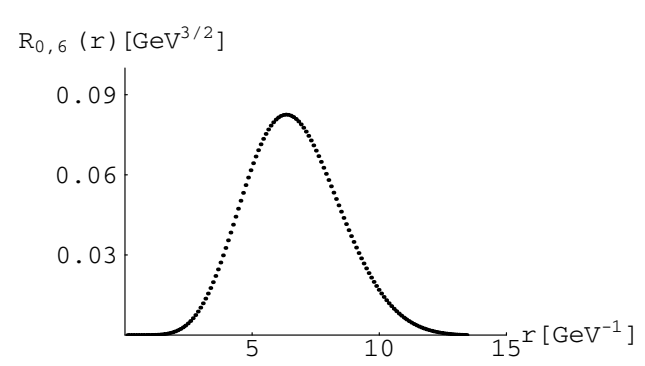

Figure 2. Example of a solution of the relativistic Schrödinger equation with the finite difference and eigenvalue method. We depict the radial part $R_{n l}(r)$ of the wavefunction for a glueball with a high angular excitation $(l=6, j=8)$. The total wavefunction is obtained multiplying the radial wavefunction by the spherical harmonic $Y_{l}^{m}(\theta, \phi)$.

Table 3. Predicted energies in GeV of charmonia, lightonia, glueballs and gluelumps as a function of $J$. We limit our study to maximal $J$ states (no radial excitations and $J=L+S_{1}+S_{2}$ ) and to energies up to $5 \mathrm{GeV}$.

\begin{tabular}{lllllllll}
\hline Family $\backslash J^{\mathrm{PC}}$ & $0^{++}$ & $1^{--}$ & $2^{++}$ & $3^{--}$ & $4^{++}$ & $5^{--}$ & $6^{++}$ & $7^{--}$ \\
\hline Charmonium & & 3.12 & 3.53 & 3.87 & 4.1 & 4.46 & 4.74 & 4.88 \\
Lightonium & & 0.77 & 1.27 & 1.68 & 2.05 & 2.40 & 2.72 & 3.04 \\
Glueball & & & 2.08 & & 3.54 & & 4.67 & \\
Gluelump & & & 4.12 & 4.88 & 5.47 & - & - & - \\
Family $\backslash J^{\mathrm{PC}}$ & $8^{++}$ & $9^{--}$ & $10^{++}$ & $11^{--}$ & $12^{++}$ & $13^{--}$ & $14^{++}$ & $15^{--}$ \\
Charmonium & 5.10 & - & - & - & - & - & - & - \\
Lightonium & 3.34 & 3.63 & 3.91 & 4.18 & 4.45 & 4.70 & 4.96 & 5.21 \\
Glueball & 5.68 & & - & & - & & - & \\
Gluelump & - & - & - & - & - & - & - & - \\
\hline
\end{tabular}

(see figure 2 for a solution obtained with finite differences). Numerically the relativistic code is only twice as slow as the non-relativistic one, because we perform two diagonalizations.

We are particularly interested in large angular momentum states, with maximal $S=S_{1}+S_{2}$ and with $J=L+S$. The maximal spin is $S=1$ for the lightonium and charmonium. The maximal spin is $S=2$ for the glueball and for the gluelump. We do not compute other spin-orbit combinations or the radial excitations, because they belong to daughter trajectories, with larger decay widths, which are harder to identify experimentally.

The possible quantum numbers of the glueball are further constrained, because the gluons are bosons. Since in a colour singlet the colour wavefunction is symmetric, and because we are only considering here the maximal $S=2$ which is also symmetric, the angular wavefunction needs also to be symmetric. Therefore the maximal $J$ trajectory starts at $J=2(L=0)$ and continues with $J=4(L=2), J=6(L=4) \cdots$.

In what concerns the gluelump, the spin of the charm and anticharm pair is not expected to affect the gluelump mass significantly. For a maximal $S$ we consider that the $c \bar{c}$ pair has spin 1 and that the total spin of the $c \bar{c} g$ system is 2 . Then the leading trajectory has $J=2(L=0), J=3(L=1), J=4(L=2) \cdots$.

We compute the energies $E_{0 l}$ (up to the first state above $5 \mathrm{GeV}$ ) and the corresponding wavefunctions $R_{0 l}(r)$. The energies are displayed in table 3. The four different trajectories, respectively for the charmonium, lightonium, glueball and hybrid, are also displayed in 


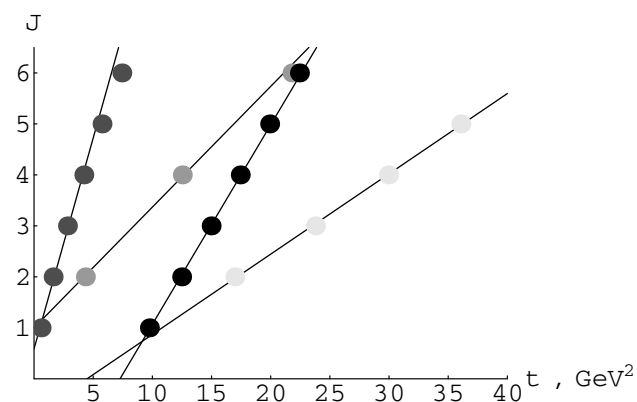

Figure 3. Chew-Frautschi plots of the leading trajectories for charmonia (black), lightonia (dark), glueballs (grey) and hybrid gluelumps (light).

Table 4. Predicted RMS $=\sqrt{\left\langle r^{2}\right\rangle}$ in $\mathrm{GeV}^{-1} \simeq 0.20 \mathrm{fm}$ of charmonia, lightonia, glueballs and gluelumps as a function of $J$.

\begin{tabular}{lllllllll}
\hline Family $\backslash J^{\mathrm{PC}}$ & $0^{++}$ & $1^{--}$ & $2^{++}$ & $3^{--}$ & $4^{++}$ & $5^{--}$ & $6^{++}$ & $7^{--}$ \\
\hline Charmonium & & 2.24 & 3.35 & 4.34 & 5.26 & 6.13 & 6.96 & 7.77 \\
Lightonium & & 2.73 & 4.06 & 5.22 & 6.28 & 7.27 & 8.21 & 9.11 \\
Glueball & & & 2.10 & & 4.06 & & 5.68 & \\
Gluelump & & & 1.86 & 2.82 & 3.64 & - & - & - \\
Family $\backslash J^{\mathrm{PC}}$ & $8^{++}$ & $9^{--}$ & $10^{++}$ & $11^{--}$ & $12^{++}$ & $13^{--}$ & $14^{++}$ & $15^{--}$ \\
Charmonium & 8.54 & - & - & - & - & - & - & - \\
Lightonium & 9.96 & 10.79 & 11.59 & 12.37 & 13.11 & 13.83 & 14.55 & 15.24 \\
Glueball & 7.13 & & - & & - & & - & \\
Gluelump & - & - & - & - & - & - & - & - \\
\hline
\end{tabular}

figure 3. The corresponding radius mean square are displayed in table 4 , where a radius of $1 \mathrm{GeV}^{-1}$ corresponds to $0.197 \mathrm{Fm}$.

We now compare our glueball trajectory with the one with the Casimir scaling. The potential $\mathcal{C}$ is refitted in the Casimir scaling case, to maintain the intercept equal to 1 . The new constant potential is $\mathcal{C}=-0.39$, and only the slope of the Chew-Frautschi trajectory changes. Our model (c), with a pair of fundamental strings, yields the trajectory

$$
j=1.00+0.24 t .
$$

In particular, the slope is close to the slope of 0.25 of the soft pomeron model of Donnachie and Landshoff [47]. The model (b) with Casimir scaling would produce the trajectory,

$$
j=1.00+0.20 t .
$$

For the same gluon masses of $0.8 \mathrm{GeV}$, model (b) with Casimir scaling would have a smaller slope, smaller than the Donnachie slope by $20 \%$.

\section{Estimating decay widths of mesons and glueballs with large $J$}

We now estimate the decay width of the predicted hadrons, and this includes several hadrons with large $J$ which have not yet been detected. Let us consider for instance the decay of a glueball $[50,51]$. We may assume that the decay is initiated when, either a gluon in the colourelectric flux tube, or a 'massive' constituent gluon, is transformed into a quark-antiquark pair with the QCD quark-gluon coupling. 


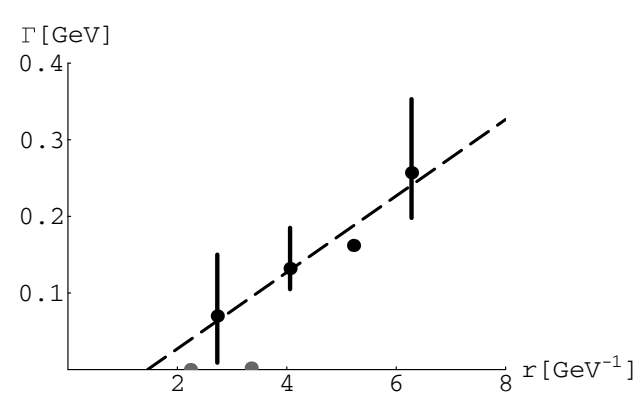

Figure 4. Decay width of angular excited resonances as a function of the theoretically estimated RMS. The decay widths are extracted from the review of particle physics [52]. The black dots are the average of the different $I=0, I=1$ and $\mathrm{K}^{*}$ lightonium decay widths. The vertical lines span from the maximum to the minimum of these lightonium decay widths. The dashed line corresponds to the best linear fit of the light data. The grey dots represent the charmonium decay widths.

Then the glueball is transformed into an excited meson or a hybrid. Because this is not a stable system, it also decays, again with the same quark-antiquark pair creation. Essentially we have a cascade of decays, because the first decay products are unstable systems. This accounts for the final decay product of several pseudoscalar mesons.

Let us consider the decay scenario where string breaking dominates over the direct constituent gluon decay. If the constituent gluon mass is lower than the constituent quarkantiquark mass, such a dominance is possible. Here $m_{g}=0.8 \mathrm{GeV} \leqslant 2 m_{q}=0.88 \mathrm{GeV}$. While the problem of the constituent gluon decay remains to be solved, here we neglect this effect. Therefore our results for the decay widths are lower bounds on the actual decay width.

In this scenario a string is cut and a $\bar{q} q$ pair is created. Each quark remains at one end of the open parts of the string. Statistically, it is plausible that the probability of producing a $\bar{q} q$ pair is proportional to the string length. It occurs that twice the string length in the glueball $g g$, is close to the string length in the $q \bar{q}$ case, with the same angular momentum. Thus, in the glueball with a double fundamental string, the total string length is similar to the string length of the light meson with the same angular momentum. So the probability of having the breaking of a string is similar in these glueballs with $J^{\mathrm{PC}}=2^{++}, 4^{++}, 6^{++} \ldots$ is respectively similar to the probability of having a string breaking in light mesons with $J^{\mathrm{PC}}=1^{--}, 3^{--}, 5^{--} \ldots$

To compute the decay widths, one would still have to evaluate the overlaps with the decay products, and the available phase space. In multiple particle products, it remains difficult, in the present state of the art of glueballs, to study quantitatively the decays. Therefore we will essentially focus into the relation of the string length with the decay width.

For an educated guess it is convenient to study the decays of conventional mesons. The decay widths extracted from the Review of Particle Physics [52] are shown in table 1. The corresponding string lengths can be approximated by the RMS of table 4. To show the dependence of the decay width in the string length, a plot is drawn in figure 4.

In figure 4 it is clear that the decay width of the light mesons is well fitted by a linear increase with the string length. Fitting the average of the different $I=0, I=1$ and $\mathrm{K}^{*}$ lightonium decay widths we get

$$
\Gamma=\gamma\left(\mathrm{RMS}-r_{0}\right) \pm \Delta \Gamma
$$

where $\gamma=0.05 \pm 0.01 \mathrm{GeV}^{2}$ and $r_{0}=1.4 \pm 0.6 \mathrm{GeV}^{-1}$. The error in the parameters $\gamma$ and $r_{0}$ are statistical errors. The error $\Delta \Gamma=0.1 \mathrm{GeV}$ includes the details of the decay processes. 
Table 5. Estimated decay widths in $\mathrm{GeV}$ of lightonia and glueballs as a function of $J$. Only the string breaking effect is considered. The statistical and systematic error bars are detailed in equation (14).

\begin{tabular}{lllllllll}
\hline Family $\backslash J^{\mathrm{PC}}$ & $0^{++}$ & $1^{--}$ & $2^{++}$ & $3^{--}$ & $4^{++}$ & $5^{--}$ & $6^{++}$ & $7^{--}$ \\
\hline Lightonium & & 0.06 & 0.13 & 0.19 & 0.24 & 0.29 & 0.34 & 0.38 \\
Glueball & & & 0.14 & & 0.33 & & 0.49 & \\
& & & & & & & & \\
Family $\backslash J^{\mathrm{PC}}$ & $8^{++}$ & $9^{--}$ & $10^{++}$ & $11^{--}$ & $12^{++}$ & $13^{--}$ & $14^{++}$ & $15^{--}$ \\
Lightonium & 0.42 & 0.46 & 0.50 & 0.54 & 0.58 & 0.61 & 0.65 & 0.68 \\
Glueball & 0.64 & & - & & - & & - & \\
\hline
\end{tabular}

If we extrapolate this linear growth to mesons with higher angular excitations, the decay width grows up to $0.68 \mathrm{GeV}$ for the $\mathrm{J}^{\mathrm{PC}}=15^{--}$meson made of light quarks, with a RMS of 15.24 $\mathrm{GeV}^{-1}$. The decay widths of the light mesons can also be extrapolated to glueballs. In this case, because the glueball has a double fundamental string, for a similar RMS, the glueball has a string length twice as long. Thus, for glueballs, the input variable RMS of equation (14) must be doubled. For instance, decay widths respectively of $0.14,0.33$ and $0.48 \mathrm{GeV}$ are expected for the first three glueballs of our series, providing the string-breaking decay process is dominant. If the direct decay of constituent gluons turns out to be important, the glueball decay widths are larger. Our predicted widths for lightonia and for glueballs are shown in table 5. Essentially, we predict that the decay widths of both lightonia and glueballs with large angular excitations are of the order of $10 \%$ of the respective masses.

However, in what concerns charmonium, the $D$ mesons are quite heavier than the light mesons. The phase space of the charmonium decay products is quite different from the phase space of the lightonium decay products, and we should not apply this simple linear rule, with the same parameters, to the charmonium decays and to the gluelump decays. Moreover, only the $1^{--}$and $2^{++}$charmonium decay widths are known, and this is not sufficient for a precise linear fit of the decay widths.

\section{Conclusion}

With a simple constituent model, we predict the masses and mean radius of charmonia, lightonia, glueballs and gluelumps. We also predict the decay widths of lightonia and glueballs. The ingredients are constituent quarks and gluons, and the fundamental $S U(3)$ chromo-electric flux tube.

We compute the spectrum and wavefunctions of hadrons with angular excitations and maximal $J$. These hadrons with no radial excitations may be easier to identify in the lattice and in the experiments. Because we construct all the hadrons with the same confining strings, we are able to extrapolate the decay widths of mesons to glueballs, in a unified framework. This model can also be applied to three-gluon glueballs [53] and other many-particle systems.

Essentially we expect the angularly excited lightonia and glueballs to have decay widths of the order of $10 \%$ of the respective masses. The largest decay width we get, for hadronic masses up to $5 \mathrm{GeV}$, are of the order of $\Gamma=0.6 \mathrm{GeV}$. Note that, because we neglected the direct decay of the constituent gluons, the actual decay widths of glueballs and hybrids may be larger than those predicted here. We nevertheless point out that the decay width $\Gamma=140 \mathrm{MeV}$ of our $2^{++}$glueball is identical to the one predicted by Cotanch and Williams with vector meson dominance [54, 55], and this suggests that our approximation, of neglecting the direct decay of the constituent gluons, is correct. 
However, the large mass of the glueballs and of the other studied hadrons enable final decay products into a large number of mesons. The first decay is expected to result in excited hadrons, and a cascade of decays into lower states is expected to produce several pions in the final state. Because the partial wave analysis may be quite difficult when, say, more than four pions are produced, new efforts in lattice QCD and in the detectors are necessary to identify the quantum numbers of the angular excited mesons, glueballs and hybrids.

\section{Acknowledgments}

PB thanks discussions on type-II superconductors with Marco Cardoso and Pedro Sacramento, on the pomeron with Barbara Clerbaux and Mike Pichowsky, on constituent gluons and glueballs with Steve Cotanch, Paola Gianotti, Felipe Llanes-Estrada, Orlando Oliveira and Nicoletta Stella, on gluelumps with Alexei Nefediev, and on excited strings with Fumiko Okiharu.

\section{References}

[1] Collins P D B 1977 An Introduction to Regge Theory and High Energy Physics (Cambridge: Cambridge University Press)

[2] Donnachie S, Dosch G, Nachtmann O and Landshoff P 2002 Pomeron Physics and QCD (Cambridge: Cambridge University Press)

[3] Simonov Y A 1990 Phys. Lett. B 249514

Kaidalov A B and Simonov Y A 2000 Phys. At. Nucl. 631428

Kaidalov A B and Simonov Y A 2000 Yad. Fiz. 631428 (Preprint hep-ph/9911291)

Kaidalov A B and Simonov Y A 2000 Phys. Lett. B 477163 (Preprint hep-ph/9912434)

[4] Llanes-Estrada F J, Cotanch S R, Bicudo P, Ribeiro J F T and Szczepaniak A 2002 Nucl. Phys. A 71045 (Preprint hep-ph/0008212)

[5] Meyer H B and Teper M 2005 Phys. Lett. B 605344

[6] Morningstar C J and Peardon M J 1999 Phys. Rev. D 60034509 (Preprint hep-lat/9901004)

[7] Liu D Q and Wu J M 2002 Mod. Phys. Lett. A 171419 (Preprint hep-lat/0105019)

[8] Bugg D V 2004 Phys. Rep. 397257 (Preprint hep-ex/0412045)

[9] Nambu Y and Jona-Lasinio G 1961 Phys. Rev. 122345 Nambu Y and Jona-Lasinio G 1961 Phys. Rev. 124246

[10] Yaouanc A Le, Oliver L, Pene O and Raynal J-C 1984 Phys. Rev. D 291233 Yaouanc A Le, Oliver L, Ono S, Pene O and Raynal J-C 1985 Phys. Rev. D 31137

[11] Adler S and Davis A C 1984 Nucl. Phys. B 224469

[12] Bicudo P and Ribeiro J E 1990 Phys. Rev. D 421611 Bicudo P and Ribeiro J E 1990 Phys. Rev. D 421625 Bicudo P and Ribeiro J E 1990 Phys. Rev. D 421635

[13] Delbourgo R and Scadron M D 1979 J. Phys. G: Nucl. Phys. 51621

[14] Bicudo P, Cotanch S, Llanes-Estrada F, Maris P, Ribeiro E and Szczepaniak A 2002 Phys. Rev. D 65076008

[15] Bicudo P 2003 Phys. Rev. C 67035201 (Preprint hep-ph/0311277)

[16] Llanes-Estrada F and Bicudo P 2003 Phys. Rev. D 68094014

[17] Nielsen H B and Olesen P 1973 Nucl. Phys. B 6145

[18] Brisudova M M, Burakovsky L, Goldman T and Szczepaniak A 2003 Phys. Rev. D 67094016 (Preprint nucl-th/0303012)

[19] Szczepaniak A P and Swanson E S 2003 Phys. Lett. B 57761 (Preprint hep-ph/0308268)

[20] Brau F and Semay C 2004 Phys. Rev. D 70014017

[21] Faddeev L, Niemi A J and Wiedner U 2004 Phys. Rev. D 70114033 (Preprint hep-ph/0308240)

[22] Meyer H B 2005 Preprint hep-lat/0508002

[23] Kalashnikova Y S 1996 Phys. At. Nucl. 591303 Kalashnikova Y S 1996 Yad. Fiz. 59N8 1363 (Preprint hep-ph/9510371)

[24] Simonov Y A 2001 Phys. At. Nucl. 641876 Simonov Y A 2001 Yad. Fiz. 641959 (Preprint hep-ph/0110033)

[25] Bernard C W 1982 Phys. Lett. B 108431 
[26] Marenzoni P, Martinelli G, Stella N and Testa M 1993 Phys. Lett. B 318511

[27] Bonnet F D R, Bowman P O, Leinweber D B and Williams A G 2000 Phys. Rev. D 62051501 (Preprint hep-lat/0002020)

[28] Silva P J and Oliveira O 2004 Nucl. Phys. B 690177 (Preprint hep-lat/0403026)

[29] Alkofer R and von Smekal L 2001 Phys. Rep. 353281 (Preprint hep-ph/0007355)

[30] Del Debbio L, Faber M, Greensite J and Olejnik S 1996 Phys. Rev. D 535891 (Preprint hep-lat/9510028)

[31] Bali G S 2000 Phys. Rev. D 62114503 (Preprint hep-lat/0006022)

[32] Semay C 2004 Eur. Phys. J. A 22353 (Preprint hep-ph/0409105)

[33] Bicudo P 2004 contribution to the PANDA Frascati workshop, Preprint hep-ph/0405223

[34] Pierre-Gilles de Gennes 1966 Superconductivity of Metals and Alloys (New York: Benjamin)

[35] Cardoso M, Bicudo P and Sacramento P D 2005 Preprint cond-mat/0509656

[36] Baker M, Ball J S and Zachariasen F 1985 Phys. Rev. D 312575

[37] Baker M, Ball J S and Zachariasen F 1991 Phys. Rep. 20973

[38] Lucha W, Schöberl F and Gromes D 1991 Phys. Rep. 200127

[39] Luscher M and Weisz P 2004 J. High Energy Phys. JHEP07(2004)014 (Preprint hep-th/0406205)

[40] Isgur N and Karl G 1978 Phys. Rev. D 184187

[41] Szczepaniak A, Swanson E S, Ji C R and Cotanch S R 1996 Phys. Rev. Lett. 762011 (Preprint hep-ph/9511422) Robertson D G, Swanson E S, Szczepaniak A P, Ji C R and Cotanch S R 1999 Phys. Rev. D 59074019 (Preprint hep-ph/9811224)

[42] Takahashi T T and Suganuma H 2004 Phys. Rev. D 70074506 (Preprint hep-lat/0409105)

[43] Juge K J, Kuti J and Morningstar C J 1998 Nucl. Phys. Proc. Suppl. 63326 (Preprint hep-lat/9709131)

[44] Bicudo P J A, Ribeiro J E F and Fernandes R 1999 Phys. Rev. C 591107 (Preprint hep-ph/9806243)

[45] Eichten E and Feinberg F 1981 Phys. Rev. D 232724

[46] Cornwall J M and Soni A 1983 Phys. Lett. B 120431

[47] Donnachie A and Landshoff P V 1998 Phys. Lett. B 437408 (Preprint hep-ph/9806344)

[48] Pelaez J R and Yndurain F J 2003 Preprint hep-ph/0312187

[49] Michael C 1985 Nucl. Phys. B 25958

Poulis G I and Trottier H D 1997 Phys. Lett. B 400358 (Preprint hep-lat/9504015)

Karl G and Paton J 1999 Phys. Rev. D 60034015 (Preprint hep-ph/9904407)

Simonov Y A 2001 Nucl. Phys. B 592350 (Preprint hep-ph/0003114)

[50] Jin H y and Zhang X m 2002 Phys. Rev. D 66057505 (Preprint hep-ph/0208120)

[51] Cotanch S R and Williams R A 2005 Phys. Lett. B 621269 (Preprint nucl-th/0505074)

[52] Eidelman S et al 2004 Phys. Lett. B 5921

[53] Llanes-Estrada F J, Bicudo P and Cotanch S R 2005 Preprint hep-ph/0507205

[54] Cotanch S R and Williams R A 2004 Phys. Rev. C 70055201 (Preprint nucl-th/0403051)

[55] Cotanch S R and Williams R A 2005 Phys. Lett. B 621269 (Preprint nucl-th/0505074) 